\title{
Self-perceived Psychophysical Well-being of Young Competitive Swimmers with Physical or Intellectual Impairment
}

Luca Puce 1,*, Lucio Marinelli ${ }^{1}$, Nicola G. Girtler ${ }^{1}$, Ilaria Pallecchi ${ }^{2}$, Laura Mori ${ }^{1}$, Marina Simonini ${ }^{3}$, Carlo Trompetto ${ }^{1}$

${ }^{1}$ Department of Neuroscience, Rehabilitation, Ophthalmology, Genetics, Maternal and Child Health, University of Genoa, Genoa, Italy

${ }^{2}$ CNR-SPIN, Physics Department, Genoa, Italy

${ }^{3}$ Struttura Complessa Recupero e Rieducazione Funzionale, ASL3 Regione Liguria, Italy

* Corresponding author: Luca Puce, L.go P. Daneo, 3 - 16132 Genoa, Italy; e-mail: luca1puce@gmail.com

\section{Abstract}

Regular practice of sport activities yields psychophysical, physical, and emotional benefits for both the general population and persons affected by physical or intellectual impairments. Practicing competitive sport may add further value to these benefits. The objective of this observational cross-sectional study was to investigate the role of competitive sport practice in enhancing self-perceived psychophysical and emotional well-being, using the Psychological General Well-Being Index (PGWBI) and the Short Form-12 (SF-12) indices. One hundred young Italian competitive swimmers affected by physical or intellectual impairment were recruited at national events. These respondents' results were compared with those of a control group of 100 Italian participants who did not practice competitive sport but who were also affected by physical or intellectual impairment, randomly selected from rehabilitation clinics and communities of young people with disabilities. Scores of psychological and emotional well-being were higher by $40 \%$ or more for the practitioners of competitive sport $(p<.0001$; Cohen's effect size $d \geq 1.3$ ). While our study's results suggest possible positive psycho-physical benefits to competitive sport practice for young people affected by physical or intellectual impairment, longitudinal research is needed to be certain that our results are not due selfselection into sports participation of persons with disabilities, who also have a uniquely higher sense of well-being.

Trial registration: ISRCTN14389453 (29 June 2017)

Keywords: Sport for People with Impairments, Young People Well-Being, Sport Psychology, Paralympic Swimming 


\section{Introduction}

The beneficial effects of regularly practiced amateur sport activities on general health, psychophysical well-being, and broad disease prevention are intensely promoted and advertised in modern society. At present, these benefits are so widely established and shared in public opinion that they have passed from scientific understanding (Eime, Young, Harvey, Charity \& Payne, 2013; Rasciute \& Downward, 2010; Scully, Kremer, Meade, Graham, \& Dudgeon, 1998) to general acceptance and undisputed lifestyle guidelines (Janssen, 2007; Services, US Department of Health and Human, 2008). Likewise, or perhaps to an even greater degree, people affected by physical or intellectual impairment can obtain psychological, social, and health benefits from participation in sport activities (Chawla, 1994; Giacobbi, Stancil, Hardin, \& Bryant, 2008; Martin Ginis, Jetha, Mack \& Hetz, 2010; Rogers, Furler, Brinks \& Darrah, 2008; Verschuren et al., 2007).

Whereas physical well-being is strictly related to the type and severity of the individual's physical impairment, psychological well-being may not be so restricted, since the sport context may offer benefits that outweigh the psychological implications of physical impairment. Psychological well-being is defined as the dynamic balance between one's personal needs and potentialities on one hand, and the characteristics of the external environment on the other hand (Dodge, Daly, Huyton \& Sanders, 2012; Felce \& Perry, 1995; Garcia, Nima, \& Kjell, 2014; Ryff \& Keyes, 1995). If sport activities are practiced at a competitive level, the related emotional, motivational, and social characteristics of that environment may provide a precious contribution to the individual psychological well-being of people affected by physical or intellectual impairment, helping them shape their own lives through a steady realization of personal objectives, favoring an acceptance of their pathology and healing the natural imbalance between their needs/potentialities and environmental demands (Ryff \& Keyes, 1995). According to prior literature, the strength of one's well-being, including personal growth, optimism, and strong social support networks, increases as athletes with impairments increase their level of competition (Macdougall, O'Halloran, Sherry, \& Shields, 2016). This is particularly crucial in youthful life stages - adolescence and young adulthood when major mental and physical changes occur and accentuate the effects of positive and negative external influences (Dykens, Rosner, \& Butterbaugh, 1998; Pollard \& Lee, 2003; Sherrill, Hinson, Gench, Kennedy, \& Low, 1990; Wilson \& Clayton 2010).

Research studies have investigated the effect of practicing competitive sport on self-concept (Dykens \& Cohen 1996; Weiss, Diamond, Demark, \& Lovald, 2003) and social competence (Dykens \& Cohen, 1996) among individuals affected by intellectual impairment. Interviews conducted with five British Paralympic swimmers aged 20-24 years with congenital impairments revealed that competitive swimming facilitates selfand social-acceptance, identity development, and a sense of normalization (Brewer, Boin, Petitpas, Van Raalte, \& Mahar, 1994). A study of 26 basketball players who used wheelchairs showed that competitive physical activity seemed to support these players' self-efficacy beliefs, feelings of empowerment and motivation for continued involvement (Giacobbi et al., 2008). A number of studies have addressed sport orientation and athletic 
identity in athletes with impairments (Martin, Adams-Mushett, \& Smith, 1995; Martin, Eklund, \& Mushet, 1997; Kokaridas, Perkos, Harbalis, \& Koltsidas, 2009; Pack, Kelly, \& Arvinen-Barrow, 2016). Athletic identity is the extent to which individuals identify with the role of an athlete, and, based on this prior research, it has been associated with the individual's self-perception, behavior, and commitment to ideological and occupational options. Martin et al. $(1995 ; 1997)$ pointed out that athletic identity involves not only positive aspects such as motivation, goal orientation, sense of empowerment, but also negative aspects such as exclusivity (i.e. inability to identify with other roles) and negative affectivity (i.e., negative emotional responses to injury, retirement or other sources). An investigation of psychophysical well-being in participants affected by either physical or intellectual impairment through the practice of competitive level sport activities should be extended into different contexts and target populations (e.g. country, type of practiced sport, age range, type of impairment, method of investigation, etc.). The interest and potential impact of this issue go well beyond the medical field and also concern ethical, social and economic aspects of our society globally. Indeed, on one hand, the well-being of individuals affected by impairments is a measure of a country's civility and inclusivity; and, on the other hand, more pragmatically but just as important, the well-being of this population may yield a significant societal reduction in healthcare costs, to which rehabilitation programs contribute remarkably.

In studies addressing current theories on well-being (Felce \& Perry, 1995; Garcia, Nima, \& Kjell, 2014; Pollard \& Lee, 2003; Ryff \& Keyes, 1995), psychological wellbeing has been multidetermined. Among the factors contributing to well-being are selfacceptance, strong and lasting affection bonds, independence, aspiration to realize objectives, and balance between self and environment. The existing literature (Brewer et al., 1994; Dykens \& Cohen 1996; Giacobbi et al., 2008; Weiss et al., 2003) is limited, but reports of results on specific dimensions of well-being in impaired competitive athletes, reveal an overall trend toward beneficial effects of engaging in competitive sport. Our objective in the current study was to assess the specific hypothesis that the practice of competitive swimming would play a positive role in the self-perceived psychophysical and emotional well-being of young participants affected by physical or intellectual impairment. In this paper, we present the results of a cross-sectional survey of the selfperceived psychophysical, physical, emotional well-being of 100 young age Paralympic athletes ${ }^{i}$ who took part in two events of the Italian calendar of Paralympic swimming competitions, namely the promotional Paralympic Swimming Italian Youth Paralympic Championship, April 29-30, 2017, Fabriano (An), Italy, and the Italian Paralympic Swimming Championship of sport clubs, May 20-21, 2017, Lignano Sabbiadoro (Ud), Italy. We compared these survey results with those of a control group comprised of 100 young participants who were also affected by physical or intellectual impairment but who did not practice competitive level sport. In this effort, we used two widespread and welldocumented survey indices, namely the Psychological General Well-Being Index (PGWBI) (Dupuy, 1984) and the 12-Item Short-Form Health Survey (SF-12; Ware,

i Throughout this work, we use the term Paralympic Swimmers to indicate those athletes who hold an Athlete's license issued by either the International Paralympic Committee IPC or the Italian Federation for Paralympic Swimming FINP, valid the for the relevant season. 
Kosinski, \& Keller, 1996), to provide a quantitative probe of self-perceived psychophysical and emotional well-being in terms of different well-being domains. Our main independent variable was the practice of competitive sport, but we also examined gender, age, the acquired or congenital nature of the participants' impairment, the type of participant disorder, and the mode of survey administration.

\section{Method}

\section{Participants and survey administration procedures}

We adopted the following participant recruitment criteria: (a) diagnosis of physical or intellectual disability, (b) 11-23 years of age, (c) self-awareness ability for responding to questionnaires with or without assistance, and (d) holding an Athlete's license issued by either the International Paralympic Committee IPC or the Italian Federation for Paralympic Swimming FINP, valid the for the relevant season. We chose criterion (b) as representative of the youth age range, according to the European Para Youth Games participation criteria and in compliance with the World Health Organization age classification groups. We evaluated criterion (c) with help from relatives and coaches, who assisted the participants in completing the questionnaires. We applied criterion (d) to the population sample under study, and an opposite criterion to the control sample, namely no practice of sport at a competitive level.

Sport practitioner participants were the young Paralympic athletes who took part in the promotional Italian Youth Paralympic Swimming Championship ("Campionati Italiani Promozionali Giovanili"), April 29-30, 2017, Fabriano (AN), Italy, and in the Italian Paralympic Swimming Championship of sport clubs ("Campionato Italiano di Società"), May 20-21, 2017, Lignano Sabbiadoro (UD), Italy. At both events, in briefings prior to the event onset, we described this study and gave detailed instructions for the administration of the questionnaires, according to related recommendations (Apolone et al., 2005). The briefings were attended by FINP executives, coaches, athletes, and relatives of the athletes. During the competitions, we distributed the questionnaires in paper form to all the participants. In addition, we edited the same questionnaires and related informative notes through Google Drive software and made them available online, through a link posted on the official FINP website (http://www.finp.it/), emphasizing the importance of the project to foster participation. We collected 195 questionnaires from participants in the competitions; out of these, 92 were rejected because they did not fulfill the 11-23 age range criterion and seven were rejected because participants affected by severe forms of autism were not able to answer properly, despite assistance from their coaches. Finally, we recruited four other participants who were practicing competitive triathlon and affected by multiple sclerosis in order to get specific comparative information on participants who were affected by severe progressive physical conditions. This process yielded 100 completed questionnaires, 58 in paper form and 42 online, were correctly completed and selected for data analysis.

We also recruited an equal number $(\mathrm{N}=100)$ of young participants affected by impairment who did not practice competitive sport to use as a control group. These 
participants were distributed within the same age range and levels of education as the sample of Paralympic athletes. Forty-seven control participants were randomly selected from local rehabilitation clinics, while the remaining 53 were randomly recruited among attendees of a promotional workshop entitled "Sport and disability in children and adolescents" that took place June 10, 2017, at the Sport Festival, Genova, Italy.

This research was approved by the local Ethics committee CER-Liguria (regional ethical committee of region Liguria, Italy), reference number 351REG2017. The participation of all participants was on a voluntary and anonymous basis. All participants, or participants' parents (in the case of participants under legal age), gave informed consent to engage in this research.

\section{Instruments}

For this research, we used two scientific survey indices - the Psychological General Well-Being Index (PGWBI) (Dupuy, 1984) and the Short Form-12 (SF-12) index (Ware et al., 1996; Ware, Kosinski, \& Keller, 1998), whose validity and reliability was demonstrated in literature (Lundgren-Nilsson, Jonsdottir, Ahlborg, \& Tennant, 2013; Gandek et al., 1998, Ware et al., 1996). Each of these have a generic (not syndromespecific) character and focus on the respondents' psychological and emotional wellbeing, rather than on their physical well-being. This is a key requirement for instruments that probe a significantly heterogeneous sample of participants who have different types of health conditions or impairments. Both indices are divided into multiple subtests or domains, that address different aspects of psychological well-being, allowing a more delineated data analysis for describing a person's health and psychophysical/emotional profile. The two questionnaires required no more than 30 minutes to complete and were able to be self-administered or administered in a face-to-face interview (best for participants with either visual or intellectual impairments). Widespread utilization of these and other similar indices has been well documented in prior literature (Becker et al., 1997; Boman, Bryman, \& Möller, 2004; Wiklund \& Karlberg, 1991).

The PGWBI questionnaire (Lundgren-Nilsson et al., 2013) consists of 22 items, each with six possible answers, whose score is proportional to the level of psychological and general well-being, rated by respondents on a six-point scale from 0 to 5 . The 22 items are sorted into six different domains: anxiety, depressed mood, positive well-being, self-control, general health, and vitality. Each domain includes 3-5 items.

The SF-12 index (Gandek et al., 1998) has a two-fold character, in that it addresses both physical and mental health measured by two separate synthetic indices, the PCS-12 and MCS-12 (physical component summary and mental component summary). This measure probes a combination of physical and mental functions and well-being, the degree of impairment on both a social and individual basis, and a personal evaluation of general health. Through this instrument we were primarily interested in the respondent's mental health, whereas we treated physical health data as ancillary information in our data analysis. This questionnaire is made up of 12 items and assesses eight domains: physical functioning, role limitations due to physical health, role limitations due to mental health, emotional well-being, bodily pain, vitality, social 
functioning, general health. The combined use of PGWBI and SF-12 indices has been previously recommended (Grossi, Mosconi, Groth, Niero, \& Apolone, 2005).

Together with the above two questionnaires, we administered a further questionnaire asking the participants to provide additional personal data and details on the administration conditions of the PGWBI and SF-12 questionnaires. Specifically, we sought to know the participants' age, gender, level of education, kind of impairment, method of administration of the questionnaires (self-administered, administered with the assistance of someone else, administered as a face-to-face interview), and place of administration of the questionnaires (e.g. at home, in a clinic, at the venue of a sport competition even, etc.). Finally we provided participants an explanatory note on the project and its scientific aim. In a protocol paper (Puce, Marinelli, Mori, Pallecchi, \& Trompetto, 2017), we reported further details on the tools used in this study.

\section{Statistical analysis}

For the statistical analysis, we used the Shapiro-Wilk test to probe the normality of the data distributions of all the parameters (age, PGWBI22, SF12-MCS, SF12-PCS). In case of a non-significant Shapiro-Wilk test, we assumed a normal data distribution. We used the Mann-Whitney test to compare the parameters between the two populations and calculated corresponding $U$ values and effect size $r=Z / \sqrt{N_{\text {tot }}}$ (where $N_{\text {tot }}$ is the total number of participants). We also calculated Cohen's effect size $d$ values for the average differences between the two populations, in the approximation of normal distributions. We set the level of statistical significance to $p<.05$ for all the tests.

\section{Results}

Table 1 illustrates participant scores on the instruments administered, grouped into the two population samples of Paralympic athletes and control participants and differentiated by gender, age, mode of survey completion (either with or without assistance), and by acquired versus congenital impairment. The same results are also displayed in Figure 1, for easy visual comparison. In the histograms of collected individual scores (not shown, available upon request), PGWBI scores were discretized in intervals of three units, whereas SF12 scores were demarked in intervals of two units, as a compromise between score resolution and sufficient number of data in each interval. On the Shapiro-Wilk test, we found that the only data sets exhibiting normal distributions were the PGWBI scores for both the Paralympic and control groups. Otherwise, a larger data sample would be necessary to obtain a normal distribution, given the large range of possible scores. Of note, the number of participants in our study was similar to past studies (Dykens \& Cohen, 1996; Lankhorst et al., 2015; Verschuren et al. 2007; Weiss et al., 2003; Wely et al., 2010; Zwinkels et al., 2015). The histograms clearly showed that the data collected from the control sample exhibited a larger variance than those of the Paralympic sample. Indeed, in Table 1, the standard deviations are almost twice as large 
as for the control sample. However, none of the data deviated by more than three standard deviations from the mean value in either population.
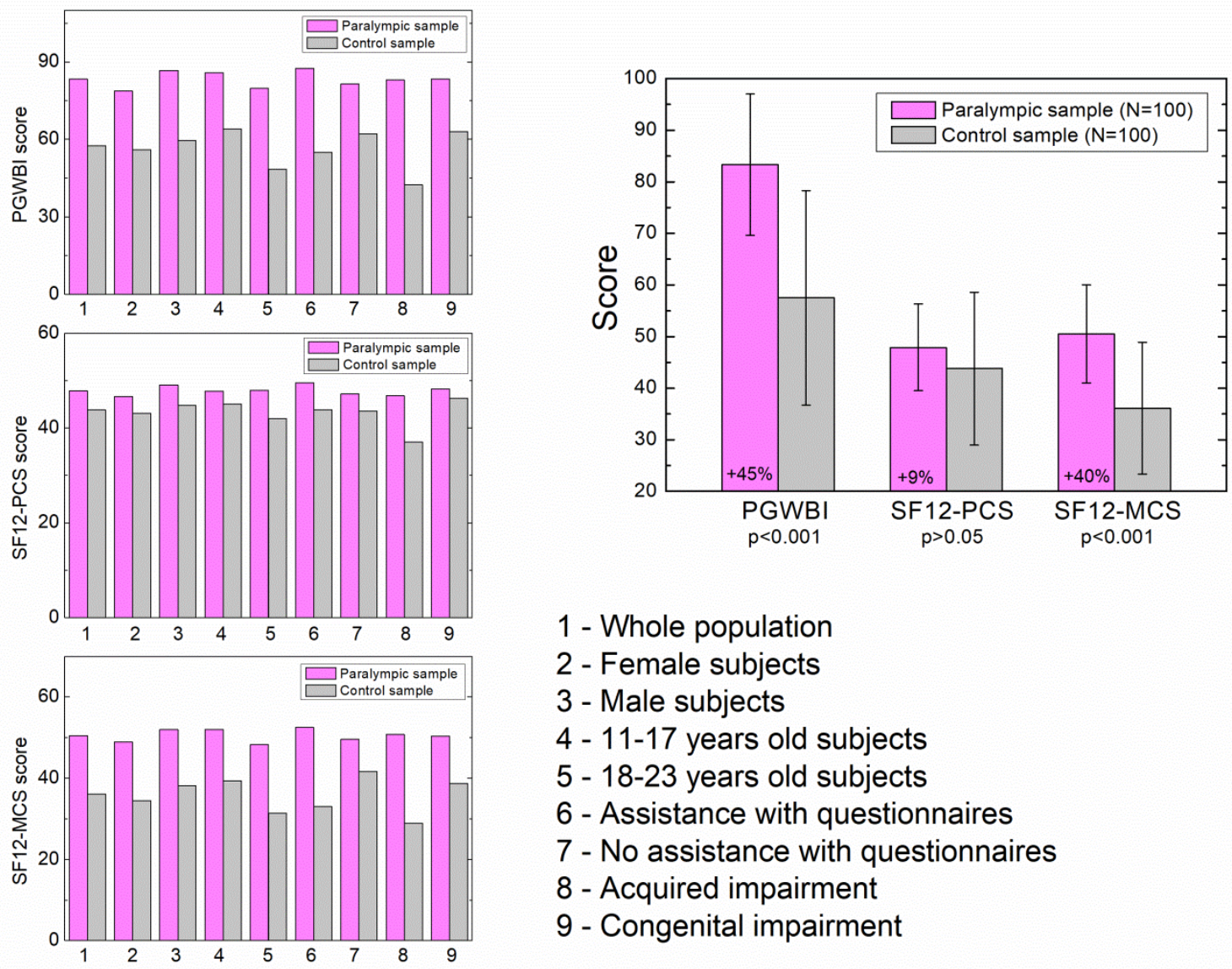

1 - Whole population

2 - Female subjects

3 - Male subjects

$4-11-17$ years old subjects

5 - 18-23 years old subjects

6 - Assistance with questionnaires

7 - No assistance with questionnaires

8 - Acquired impairment

9 - Congenital impairment

Figure 1. Well-being indices scores of the whole populations and differentiated by gender, age, mode of filling and acquired/congenital impairment. Error bars indicate standard deviations of the scores of the whole populations. PGWBI = Psychological General Well-Being Index; SF12-PCS = Short Form-12 physical component summary; SF12-MCS = Short Form-12 mental component summary. 
Table 1: Scores of the questionnaires of the Paralympic athlete and contol populations.

\begin{tabular}{|c|c|c|c|c|c|c|c|}
\hline \multicolumn{8}{|c|}{ Sample of Paralympic athletes } \\
\hline \multirow{2}{*}{ Sample } & \multirow{2}{*}{$\mathbf{N}$} & \multicolumn{2}{|c|}{ PGWBI } & \multicolumn{2}{|c|}{ SF12-PCS } & \multicolumn{2}{|c|}{ SF12-MCS } \\
\hline & & $M$ & $S D$ & $M$ & $S D$ & $M$ & $S D$ \\
\hline $\begin{array}{c}\text { whole } \\
\text { population }\end{array}$ & 100 & 83.3 & 13.7 & 47.9 & 8.4 & 50.5 & 9.5 \\
\hline female subjects & 49 & 78.7 & 13.1 & 46.7 & 8.8 & 48.9 & 9.9 \\
\hline male subjects & 51 & 86.7 & 12.8 & 49.1 & 7.9 & 52.0 & 9.0 \\
\hline $\begin{array}{c}\text { subjects aged 11- } \\
17\end{array}$ & 58 & 85.8 & 13.5 & 47.8 & 8.6 & 52.0 & 9.0 \\
\hline $\begin{array}{c}\text { subjects aged 18- } \\
23\end{array}$ & 42 & 79.7 & 13.3 & 48.0 & 8.2 & 48.3 & 9.9 \\
\hline $\begin{array}{l}\text { questionnaire } \\
\text { filled with } \\
\text { assistance } \\
\text { questionnaire }\end{array}$ & 30 & 87.5 & 13.1 & 49.6 & 7.2 & 52.5 & 6.9 \\
\hline $\begin{array}{l}\text { filled without } \\
\text { assistance }\end{array}$ & 70 & 81.5 & 13.6 & 47.2 & 8.8 & 49.6 & 10.4 \\
\hline $\begin{array}{l}\text { acquired } \\
\text { impairment }\end{array}$ & 24 & 83.0 & 14.2 & 46.9 & 8.8 & 50.8 & 10.4 \\
\hline $\begin{array}{c}\text { congenital } \\
\text { impairment }\end{array}$ & 76 & 83.4 & 13.6 & 48.2 & 8.3 & 50.4 & 9.3 \\
\hline \multicolumn{8}{|c|}{ Control sample of subjects affected by impairment, which do not practice competitive sport } \\
\hline \multirow{2}{*}{ Sample } & \multirow{2}{*}{$\mathbf{N}$} & \multicolumn{2}{|c|}{ PGWBI } & \multicolumn{2}{|c|}{ SF12-PCS } & \multicolumn{2}{|c|}{ SF12-MCS } \\
\hline & & $M$ & $S D$ & $M$ & SD & $M$ & SD \\
\hline $\begin{array}{c}\text { whole } \\
\text { population }\end{array}$ & 100 & 57.5 & 20.8 & 43.8 & 14.8 & 36.1 & 12.8 \\
\hline female subjects & 58 & 56.0 & 21.3 & 43.1 & 14.5 & 34.5 & 13.1 \\
\hline male subjects & 42 & 59.6 & 20.2 & 44.8 & 15.4 & 38.2 & 12.2 \\
\hline $\begin{array}{l}\text { subjects aged 11- } \\
\qquad 17\end{array}$ & 58 & 64.0 & 17.9 & 45.1 & 14.4 & 39.4 & 12.9 \\
\hline $\begin{array}{c}\text { subjects aged 18- } \\
23 \\
\text { questionnaire }\end{array}$ & 42 & 48.5 & 21.5 & 42.0 & 15.4 & 31.4 & 11.1 \\
\hline $\begin{array}{c}\text { filled with } \\
\text { assistance } \\
\text { questionnaire }\end{array}$ & 65 & 55.0 & 20.7 & 43.9 & 15.5 & 33.0 & 12.6 \\
\hline $\begin{array}{l}\text { filled without } \\
\text { assistance }\end{array}$ & 35 & 62.1 & 20.6 & 43.6 & 13.8 & 41.7 & 11.3 \\
\hline $\begin{array}{c}\text { acquired } \\
\text { impairment }\end{array}$ & 27 & 42.4 & 20.9 & 37.0 & 13.9 & 29.0 & 9.3 \\
\hline $\begin{array}{c}\text { congenital } \\
\text { impairment }\end{array}$ & 73 & 63.1 & 18.0 & 46.3 & 14.5 & 38.7 & 13.0 \\
\hline
\end{tabular}


For data collected from the additional questionnaires, we examined the possibility that our results could be biased due to uneven distributions of the respondents' personal data or mode of administration in the two samples, and we verified that the two populations were similar with regard to gender (49 females and 51 males in the Paralympic sample, compared to 58 females and 42 males in the control sample), age ( $M$ age $($ years $)=17.4, S D=3.7$ in the Paralympic sample; $M$ age $=17.0, S D=3.7$ in the control sample), level of education, and health condition. We registered an imbalance for the mode of questionnaire administration (with 70\% self-administered and $30 \%$ administered with assistance in the Paralympic sample and 35\% self-administered and $65 \%$ administered with assistance in the control sample) and for the setting in which participants lived (79\% urban and 21\% rural in the Paralympic sample, compared to 55\% urban and $45 \%$ rural in the control sample), the latter outcome possibly due to the fact that practicing sport on a daily basis is logistically easier in urban settings. Importantly, the two populations were homogeneously distributed by type of disorder, as shown in Figure 2. There was no statistical difference in the number of participants for any of the subgroups.

\section{Subject count}

\section{Paralympic sample}

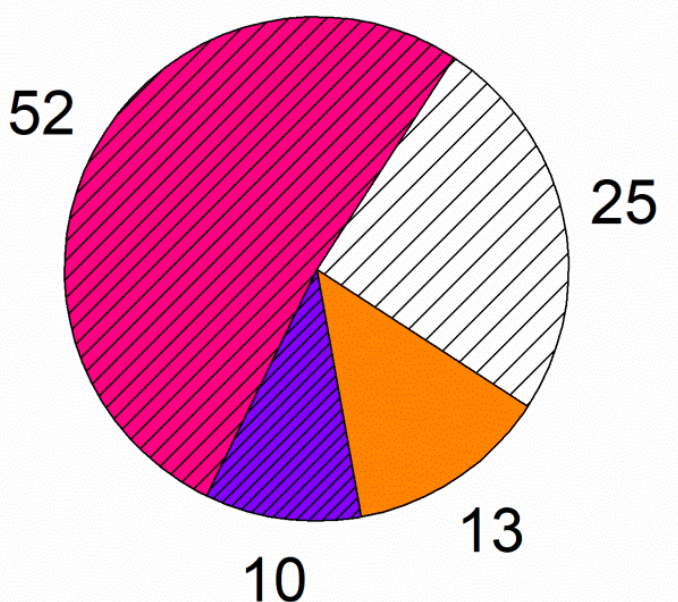

Control sample

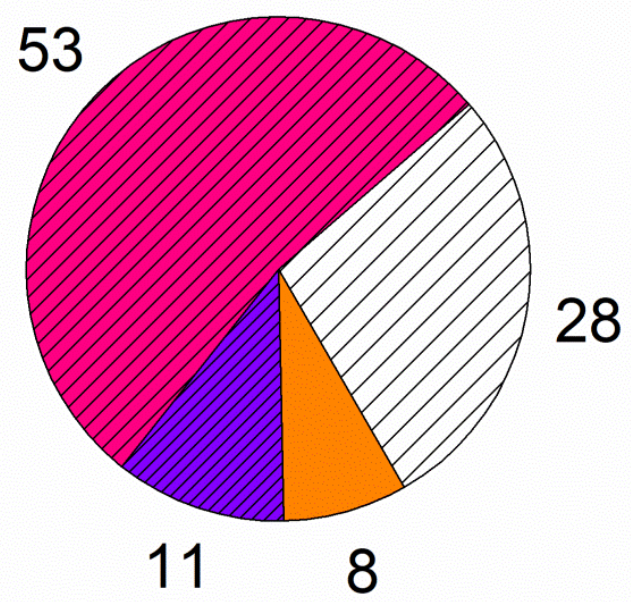

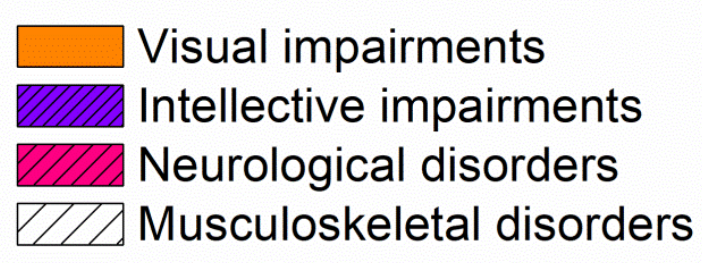

Figure 2. Pie charts of the distributions of disorders in the two populations. Numerical labels indicate the subject count in each subgroup. 
Our main finding was that the Paralympic sample scores on the PGWBI and SF12 were higher (in the direction of greater respondent well-being) than were the scores on these instruments from the control sample:

- PGWBI $(U=1554.5, p<.0001, r=.595, d=1.5)$

- SF12-PCS $\quad(U=4254.5, r=.129, d=0.3)$

- $\mathrm{SF} 12-\mathrm{MCS} \quad(U=1977.5, p<.0001, r=.522, d=1.3)$

We calculated the relative percent differences between the Paralympic and control samples and between homologous subgroups of the Paralympic and control samples with respect to the values of the control sample, whereas for subgroups of the same sample (e.g., Paralympic male versus Paralympic female or Paralympic aged 11-17 versus Paralympic aged 18-23) we calculated the relative percent differences with respect to the intermediate values among the two subgroups. The scores of the Paralympic group were higher than those of the control group by $45 \%$ for PGWBI, $40 \%$ for SF $12-M C S$ and $9 \%$ for SF12-PCS. Hence our results indicate that for the PGWBI and SF12-MCS indices, that assess the respondents' psychological and emotional well-being, the athlete sample obtained larger scores with statistical significance $(p<.0001)$. Of note, for the PGWBI and SF12-MCS indices, the corresponding Cohen's effect size $d$-values were very large $(d>1.2)$, according to Sawilowsky (2009) and the Mann-Whitney effect size $r$-values were large $(r>05)$.

Regarding the analysis of homologous subgroups of the two samples (males, females, aged 11-17 years, aged 18-23 years, with acquired impairment, with congenital impairment, who filled questionnaires with assistance, who filled questionnaires without assistance), the PGWBI and SF12-MCS score differences between all Paralympic and respective control subgroups reached statistical significance $(p<.0001$ for the subgroups with acquired impairment and $p<.00001$ for all the other subgroups). On the contrary, the SF12-PCS score differences between Paralympic and respective control subgroups were not statistically significant. The Cohen's $d$-values were very large for the PGWBI score differences between all Paralympic and respective control subgroups and even huge $(d>2.0$, Sawilowsky, 2009) in the case of Paralympic and control subgroups with acquired impairment. The Mann-Whitney effect size $r$-values for the differences in PGWBI and SF12-MCS scores were large $(r>0.5)$ between all subgroups, except for the medium $r$ effect size of the subgroups who filled the questionnaires with assistance $(0.3<$ $r<0.5)$.

Regarding the differences between subgroups of the Paralympic sample, we found statistical significance only for the PGWBI scores of males versus females subgroups, aged 11-17 versus aged 18-23 subgroups and subgroups of athletes who filled with assistance versus those who filled without assistance, but in all these cases with small $r$ effect size $(0.1<r<0.3)$. In the case of the subgroups of the control sample, we found statistical significance only for the PGWBI and SF12-MCS scores of aged 11-17 versus aged 18-23 participants and for SF12-MCS scores of participants who filled with assistance versus those who filled without assistance, with medium $r$ effect size $(0.3<r<$ $0.5)$ in all these cases. 
We now address quantitative comparisons of survey scores of different intrasample and inter-sample subgroups:

(a) For both populations, males reported 5-10\% higher well-being scores than females. Specifically, in the Paralympic sample male (relative to female) scores were higher on the PGWBI by $10 \%$, the SF12-PCS by $5 \%$, the SF $12-M C S$ by $6 \%$; in the control sample, male (relative to female scores) were higher on the PGWBI by $6 \%$, the SF12-PCS by $4 \%$, and the SF12-MCS by $10 \%$. However, statistical significance between gender subgroups within the same sample was found only for the PGWBI index in the Paralympic sample $(U=832, p=.0040, r=.288, d=0.62)$.

(b) For both populations, younger participants, aged 11-17 years, reported higher psychological and emotional well-being than older participants, aged 18-23. For the Paralympic sample, younger participants scored higher than older participants by $7 \%$ on the PGWBI, and by $7 \%$ on the SF12-MCS, but were not different $(-0.4 \%)$ on the SF-12PCS. For the control sample, younger participants scored higher than older participants by $28 \%$ on the PGWBI, $23 \%$ on the SF12-MCS, and $7 \%$ on the SF12-PCS. Statistical significance between age subgroups within the same sample was found only for the PGWBI index in the Paralympic sample $(U=879, p=.0183, r=.236, d=0.46)$ and for the PGWBI $(U=735.5, p=.00076, r=.337, d=0.79)$ and SF12-MCS $(U=786.5, p$ $=.0026, r=.301, d=0.66)$ indices of the control sample. Among the comparison between the homologous age subgroups of the two samples, the largest difference was found in the PGWBI scores of the participants aged 18-23, that were $64 \%$ higher in the Paralympic group than in the control group $(U=175, p<.00001, r=.690, d=1.74)$.

(c) Regarding assistance in completing questionnaires, for participants in the Paralympic sample, those using assistance (relative to those relying on self-administered questionnaires) were larger by $7 \%$ for the PGWBI, $6 \%$ for the SF-12-MCS, and $5 \%$ for the SF12-PICS, with statistical significance only for the PGWBI index $(U=783.5, p=$ $0.046, r=.200, d=0.45)$. For control participants, the opposite was true, in that those relying on self-administered questionnaires were a higher number on the PGWBI by $12 \%$, the SF $12-\mathrm{MCS}$ by $23 \%$, and were equivalent on the SF12-PCS (higher by only $0.7 \%)$, with statistical significance only for the SF12-MCS index $(U=675, p=.00084, r$ $=.334, d=0.72$ ).

(d) Regarding whether the nature of the respondents' impairment (i.e., acquired or congenital) affected survey results, there was a much greater influence among control participants, while Paralympic participants with either congenital or acquired impairments responded similarly. Control participants who reported acquired impairment (versus congenital) impairments scored higher by $96 \%$ on PGWBI, 75\% on SF12-MCS, and $27 \%$ on SF12-PCS. However, these intra-sample subgroup differences were not statistically significant. Among inter-sample comparison of homologous impairment type subgroups, the largest difference was in the PGWBI scores of the participants with acquired impairment, that were higher in the Paralympic group by $96 \%$, with statistical significance $(U=121.5, p=.00014, r=.690, d=2.25)$. 
(e) Partial scores on the survey instruments for selected PGWBI domains are shown in Figure 3. The values for the Paralympic sample (compared to the control sample) were all in the direction of statistically significant better physical and psychological well-being with specific subtest scores as follows: positive well-being of $13.54 / 20$ (13.54 out of a maximum score 20 for this domain) higher by $71 \%(U=1581, p$ $<.00001, r=.591, d=1.46)$; anxiety of $18.94 / 25$ higher (i.e., less anxiety ) by $31 \%(U=$ $2461, p<.00001, r=.439, d=0.96$ ); depressed mood of 12.92/15 higher (i.e., less depressed mood) by 59\% $(U=1616, p<.00001, r=.585, d=1.46)$; vitality of $14.18 / 20$, higher by $39 \%(U=2383, p<.00001, r=.452, d=1.03)$; self-control of $11.47 / 15$, higher by $62 \%(U=1614.5, p<.00001, r=.585, d=1.46)$; and general health of $12.40 / 15$, higher by $28 \%(U=2768.5, p<.00001, r=.385, d=0.89)$. Hereby, focusing on selected domains relevant to mental well-being, considered particularly relevant to the International Paralympic Committee's (IPC) mission, Paralympic participants' (versus control participants') scores for positive well-being (PGWBI items 1, 9, 15 and 20), anxiety (PGWBI items 5, 8, 17, 19, 22), depressed mood (PGWBI items 3, 7, 11), vitality (PGWBI items 6, 12, 16, 21), the Paralympic were higher by $30-70 \%$.

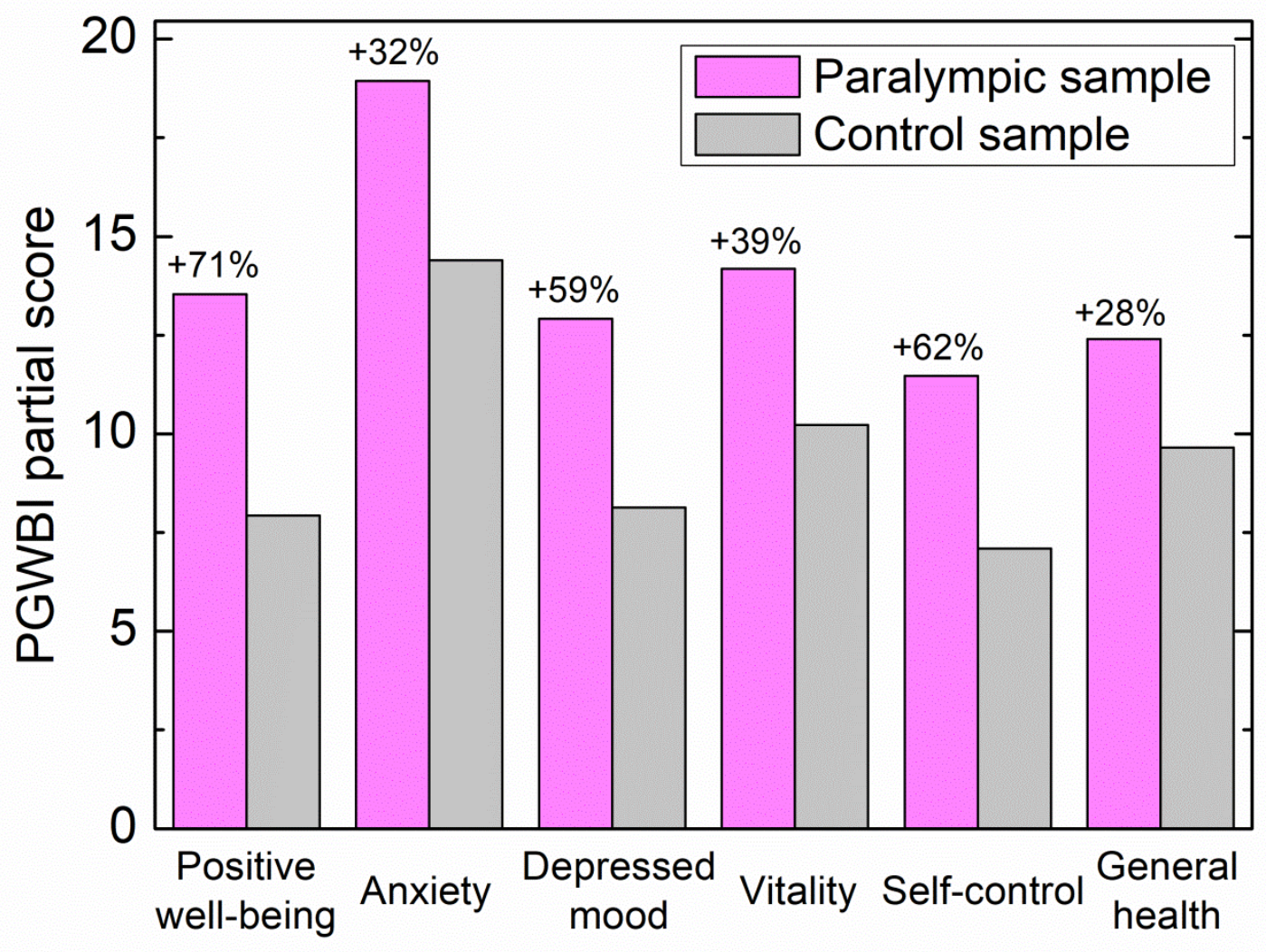

Figure 3. Partial scores of selected domains in the two populations. 
Among participants in the Paralympic sample there was no evidence of any specific health condition for which systematically higher or lower scores were registered. On the other hand, in the control population, the scores of the six participants who were affected by multiple sclerosis (MS) were systematically lowest, especially PGWBI scores, but also SF12-MCS scores. While the number of participants in each impairment subsample (i.e. 10) was too small to test whether this finding was statistically significant, these very low scores merit specific mention. For the control sample, the average PGWBI score of MS participants was five times lower than the average PGWBI score of the whole population (-413\%), and the average SF12-PCS and SF12-MCS scores were lower by as much as $-53 \%$ and $-98 \%$, respectively. Within the Paralympic sample the scores of the MS population were also lower than average, though they were significantly higher than the scores of MS participants in the control group, with the PGWBI score higher among Paralympic MS respondents by $84 \%$, the SF $12-$ MCS score higher by $65 \%$ and the SF12-PCS score higher by $18 \%$.

\section{Discussion}

The key result of this investigation was that physical and psychological wellbeing indices, particularly the PGWBI and the SF12-MCS addressing psychological and emotional well-being, were larger by $40 \%$ or more for participants with impairments who practice competitive sport relative to participants with impairments who do not practice competitive sport. This finding supported our original hypothesis. Focusing particularly on domains that assessed positive well-being, anxiety, depressed mood and vitality, we found the scores of Paralympic participants to be higher by $30-70 \%$, relative to those of other young people with physical and intellectual impairment who were not practicing competitive sport. On the contrary, the difference on the SF12-PCS scores between the two samples was not statistically significant. We attribute the latter finding to the large heterogeneity of the impairment types in the two populations, which makes comparison of self-perceived physical well-being less meaningful. Indeed, this work is rather focused on the psychological and emotional well-being described by the PGWBI and SF12-MCS indices, yet reporting all results so as to be complete.

There are a number of possible social and psychological mechanisms triggered by the practice of competitive sport (we consider competitive swimming as representative of competitive sport in general) that may contribute to an improved self-perceived wellbeing, a motivated attitude and reduced anxiety and depressive symptoms. Some mechanisms are strictly related to the physical benefits of sport activities. Sport that is adapted to a physical condition is an actual therapy that can be prescribed on a daily basis (Puce et al., 2018). Indeed, daily training sessions carried out to improve one's performance are not only aimed toward winning medals but are also medical treatment, either direct (e.g., intended to increase proportions of connective tissue in targeted muscles) or indirect (e.g., , intended to inhibit negative secondary effects of the pathology) (Jarvinen, Jozsa, Kannus, Jarvinen, \& Jarvinen, 2002; Trompetto et al., 2014). In addition, intensive sport training maximizes residual motor skills, develops new skills and improves individual kinesthetic perception, all of which may be helpful in the everyday activities of people affected by various impairments. There are also purely 
psychological, social and motivational mechanisms unique to competitive sport practice that may be beneficial to self-perceived well-being. It is well known that regular physical activity in adults, children and adolescents improves health and reduces mood disorders (Eime et al., 2013). Also, in people affected by impairments, physical activity reduces anxiety and depression and increases self-esteem and self-efficacy (Shephard, 1991). However, competitive physical activity can lead to a slight increase in stress and anxiety if there is excessive environmental pressure (Nieman, 2002).

Mental well-being includes several factors, such as optimism, self-esteem, having a life purpose, a sense of belonging and building strong and positive relationships with others. Self-esteem is a key factor in psychological well-being, and it reflects a belief and confidence in one's own ability and value. This concept is fundamental for sports players. Indeed, Fox and Corbin (1989) described self-esteem in sport as a multi-dimensional concept consisting of strength, conditioning, bodily attractiveness, and sporting competence. However, some researchers suggest that people affected by impairments may have lower self-esteem than able-bodied people, and Bredahl (2013) found that 75\% of adults with impairment had negative experiences in sport. By contrast, adolescents with intellectual impairment taking part in swimming programs showed significant improvements in their sporting self-esteem when they were competing against others in their own impairment group (Wright \& Cowden, 1986). Similarly, in our sample, increased sports self-esteem may have been due to the fact that these individuals were competing against others in their own impairment group, highlighting the importance of belonging to a peer group that shares one's experiences.

In sport events, a peculiar feeling is created in which the competitive spirit overcomes any tendency to commiserate over the physical or intellectual conditions of the athletes. This agonistic attitude may cancel out stereotypical responses to the pathology (Pack et al., 2016) and encourages overcoming one's limits. Indeed, athletes with impairments have comparable levels of athletic identity to athletes who do not have any impairment, and they compete in sport at similar levels of intensity (Groff \& Zabriskie, 2006; Martin et al., 1995). The practice of competitive sport shifts the focus from impairment to competency and proficiency at a given activity (Pack et al., 2016). Within Paralympics, any losing athlete is beaten by a peer who is affected by similar impairment; hence any excuse for defeat is unacceptable and is rather replaced by selfdirection toward stronger dedication and effort and a willing motivation to improve (Giacobbi et al., 2008; Kokaridas et al., 2009; Martin et al., 1995). On the opposite side, among participants who do not practice competitive sport, impairments are more likely to cause a depressed mood. Among our participants, neuro-progressive syndromes such as MS were associated with strongly depressed psychological conditions.

Regarding possible medical and economic implications of our findings of improved psychophysical fitness among competitive sport athletes affected by impairments, rehabilitation effectiveness of sport-related training sessions may be superior to typical weekly rehabilitation training, not only physically (Runciman , Derman, Ferreira, Albertus-Kajee, \& Tucker, 2015; Runciman, Tucker, Ferreira, Albertus-Kajee, \& Derman, 2016), but also emotionally and psychologically. For example, structuring rehabilitation programs according to periods targeted at improving 
medium- and long-term physical performances and scheduling collective performance tests, as in the case of competitive training, could have a huge motivational role.

Effectiveness of such novel rehabilitation programs would leverage on the ability of the impaired participants to take advantage of the specificities of their body and get motivated by pursuing and sharing personal goals, with benefits in different aspects of life. Moreover, transferring knowledge about training load parameters such as volume, intensity and recovery times, properly adapted to the type and severity of individual impairments, from the competitive sport field to the rehabilitation protocols could yield significant benefit to psychophysical well-being. As a consequence, the health care system may benefit from promoting Paralympic sport participation not only from a medical point of view but also from an economic perspective, as significant rehabilitative cost savings are likely. Indeed, enhanced psychophysical condition favours improved autonomy in daily activities and helps tackling negative effects of progressive and nonprogressive disorders, both of which could help saving costs of the health care system.

Considering secondary outcomes of our data, as shown in Table 1, the much larger variance of survey responses among participants in our control versus Paralympic population suggests that there was a common mindset among Paralympic athletes, possibly related to the fact that they share the same community, lifestyles and ambitions. Perhaps this occurred because 22 of the 100 Paralympic participants completed the survey at the venue of a competitive event and were surrounded by peers at the time. In contrast, all the control participants completed questionnaires online, probably alone. Past researchers showed that the mode of questionnaire administration can affect the obtained scores (Bowling, 2005; Grossi et al., 2005; Gundy \& Aaronson, 2010; Hood et al., 2012; Weinberger, Oddone, Samsa, \& Landsman, 1996). Furthermore, as $\sim 20 \%$ of the of the Paralympic group completed the questionnaire during the event, their responses may have been influenced by higher than normal levels of endorphins that are known to improve mood during or after acute exercise (Dishman \& O'Connor, 2009). Endorphins modulate dopamine neurons involved in motivation, pleasure, and the reward system to enhance moods indirectly.

We found that assistance in the administration of the questionnaires played only a minor role, if any, in the respondents' answers, consistent with findings in other studies (Puhan, Ahuja, Van Natta, Ackatz, \& Meinert, 2011; Ramos-Jorge et al., 2012; Rutherford, Nixon, Brown, Lamping, \& Cano, 2014; Tsakos, Bernabé, O’Brien, Sheiham, \& de Oliveira, 2008). Among participants who had help completing questionnaires in our study (relative to those who completed questionnaires without help), scores of Paralympic participants were $6 \%$ higher while those of control participants were around $-10 \%$ lower. However, it is worth noting that there an interesting imbalance in the numbers of self-administered and administered-withassistance questionnaires between the Paralympic and control samples, namely a larger fraction of questionnaires were administered with assistance in the control sample. This possibly suggests greater impairment in the control group, which may have influenced the self-perception of well-being.

Regarding gender, we observed that males' self-perceived well-being scores were $5-10 \%$ higher than females'. However, because this finding was evident for both 
Paralympic and control participants and was consistent with past findings (Gestsdottir et al., 2015; Harada et al., 2008; Li, Kao, \& Wu 2015), this observation is beyond the scope of our study and does not warrant extensive discussion here. Similarly, our finding that younger participants, aged 11-17 years, perceived higher psychological and emotional well-being than older participants, aged 18-23 year, applied to both populations. However, this finding was accentuated among control participants, mainly due to older participants' differences between the Paralympic and control groups $(64 \%$ difference in PGWBI scores, $p<.0001)$. It seems that, with increasing age, there is a stronger awareness and sense of either goal achievement or goal discrepancy (Cheng, 2004; Nilsson, Leppert, Simonsson, \& Starrin, 2010) and that this contributes to reinforce both positive and negatives among older participants.

It is worth noting that among control participants, congenital (versus acquired) impairment was associated with higher well-being scores. This may be due to the greater difficulty accepting and adapting to a later occurring impairment condition. Yet, this difficulty seemed to be attenuated by the competitive sport experience of participants in the Paralympic group in which scores from those with congenital and acquired impairments were similar. The difference between PGWBI scores of Paralympic and control acquired impairment subgroups was as large as $96 \%(p=.00014)$.

Among the limitations of this study were the size and geographical constraints of the two participant populations, perhaps limiting the study's generalizability. Similarly, while competitive swimming is considered representative of competitive sport generally, future studies might investigate these variables among participants in other competitive sports, perhaps comparing team and individual sports. Another limitation of the study could be related to the sample size; indeed, high variance values of sample scores may indicate that that a larger sample size is needed to enhance the statistical power of the instrument. Specifically for the case of participants affected by severe and progressive syndromes such as MS, further investigations over larger numbers could confirm the dramatic effectiveness of practicing competitive sport on mental well-being, found in this study. Additionally, this study relied entirely on participants' self-reported physical and psychological well-being; future studies might extend this research paradigm by seeking input regarding the participants' well-being by surveying others who know them well. Finally, among the most important challenges to our research is the possibility that our Paralympic participants' positive attitudes developed independent from their competitive sport engagement or that they may have contributed to this group having self-selected competitive sport practice As this was a cross-sectional research design, we can not be certain of a causal direction from sport practice to higher well-being scores. Longitudinal research is needed to affirm that assumption.

\section{Conclusions}

In this study of 100 Paralympic and 100 control participants with physical and intellectual impairment conditions, the self-reported psychological and emotional wellbeing was higher by about $40 \%$ among the group who regularly practiced competitive sport. This finding was particularly strong for positive well-being, anxiety, depressed 
mood, and vitality, for which Paralympic participants scored higher by $30-70 \%$. We also found that the higher self-perceived well-being of the Paralympic group as compared to the control group increased with older (i.e., over 18 years) versus younger participants Also, somewhat remarkably, among Paralympic participants there were similar wellbeing reports for those with congenital and acquired impairments, while among control participants, perceived well-being was lower among those with acquired conditions. Thus, sport participation seemed to attenuate the negative impact of acquired impairments.

We suggest some possible social and psychological mechanisms triggered by the practice of competitive sport, that may contribute to the improvement of the selfperceived well-being and motivated attitude (Eime et al., 2013; Shephard, 1991; Fox and Corbin, 1989; Wright \& Cowen, 1986; Pack et al., 2016, Giacobbi et al., 2008; Kokaridas et al., 2009; Martin et al., 1995; Fleisher \& Zames, 2001), namely motivation toward objectives, self-commitment as opposed to self-pity, acceptance of defeat, resilience, scarce liability to depression, propensity to mutual assistance, development of longlasting relationships.

We believe that all these factors have a direct impact on psychophysical wellbeing and general health of young people affected by impairments, making our results worthy of consideration in designing rehabilitations programs. These programs could be structured in training period cycles targeted at medium- and long-terms scheduled physical performance tests, possibly organized in groups, thus providing a motivational and social added value. Moreover, they could include high intensity training sessions, that are properly adapted to the type and severity of disorder. These results could have societal benefit through promoting inclusivity and the expression of the full potential of individuals with disabilities and through healthcare cost savings. In perspective, we plan to carry out a similar study on Paralympic athletes who practice different sports, in order to compare well-being scores between athletes of different sports (more or less dynamic) and between athletes of team versus individual sports.

Declaration of conflicting interests: The Authors declare that there is no conflict of interest

Funding: The author(s) received no financial support for the research, authorship, and/or publication of this article.

\section{References}

Apolone, G., Mosconi, P., Quattrociocchi, L., Gianicolo E. A. L., Groth, N., \& Ware, J. E. Jr. (2005). Questionario sullo stato di salute SF-12, Versione Italiana (Versione IRFMN Aggiornata 2005). Milan: Istituto di Ricerche Farmacologiche Mario Negri, Milano. 
Becker, N., Bondegaard Thomsen, A., Olsen, A. K., Sjøgren, P., Bech, P., \& Eriksen, J. (1997). Pain epidemiology and health related quality of life in chronic non-malignant pain patients referred to a Danish multidisciplinary pain center. Pain, 73(3), 393400.

Boman, U. W., Bryman, I., \& Möller, A. (2004). Psychological well-being in women with Turner syndrome: somatic and social correlates. Journal of Psychosomatic Obstetrics \& Gynecology, 25(3-4), 211-19.

Bowling, A. (2005). Mode of questionnaire administration can have serious effects on data quality. Journal of Public Health, 27(3), 281-91. doi:10.1093/pubmed/fdi031.

Bredahl, A. M., (2013). Sitting and watching the others being active: the experienced difficulties in PE when having a disability. Adapted Physical Activity Quarterly, 30(1), 40-58. doi:10.1123/apaq.30.1.40

Brewer, B. W., Boin, P. D., Petitpas, A. J., Van Raalte, J. L., \& Mahar, M. T. (1994). Dimensions of athletic identity (presented at the $101^{\text {st }}$ American Psychological Association Annual Conference, Toronto, Canada). American Psychologist, 49(7), 586-635. doi:10.1037/0003-066X.49.7.586

Chawla, J. C. (1994). ABC of sports medicine: sport for people with disability. BMJ, 308, 1500. doi:10.1136/bmj.308.6942.1500.

Cheng, S.-T. (2004). Age and subjective well-being revisited: a discrepancy perspective. Psychology and Aging, 19(3), 409-15. doi:10.1037/0882-7974.19.3.409.

Dishman, R. K., \& O’Connor, P. J. (2009). Lessons in exercise neurobiology: the case of endorphins. Mental Health and Physical Activity, 2, 4-9. doi:10.1016/j.mhpa.2009.01.002.

Dodge, R., Daly, A., Huyton, J., \& Sanders, L. (2012). The challenge of defining wellbeing. International Journal of Wellbeing, 2(3), 222-35. doi:10.5502/ijw.v2i3.4. 
Dupuy, H. J. (1984). The Psychological General Well-Being (PGWB) Index. In N. K. Wenger, M. E. Mattson, C. D. Furburg, \& J. Elinson (Eds.), Assessment of Quality of Life in Clinical Trials of Cardiovascular Therapies (pp. 170-183). New York, NY: Le Jacq Publishing.

Dykens, E. M., \& Cohen, D. J. (1996). Effects of Special Olympics International on social competence in persons with mental retardation. Journal of the American Academy of Child and Adolescent Psychiatry, 35(2), 223-29. doi:10.1097/00004583199602000-00016.

Dykens, E. M., Rosner, B. A., \& Butterbaugh, G. (1998). Exercise and sports in children and adolescents with developmental disabilities. positive physical and psychosocial effects. Child \& Adolescent Psychiatric Clinics of North America, 7(4), 757-71.

Eime, R. M., Young, J. A., Harvey, J. T., Charity, M. J., \& Payne, W. R. (2013). A systematic review of the psychological and social benefits of participation in sport for children and adolescents: informing development of a conceptual model of health through sport. International Journal of Behavioral Nutrition and Physical Activity, 10, 98. doi:10.1186/1479-5868-10-98.

Felce, D., \& Perry, J. (1995). Quality of life: its definition and measurement. Research in Developmental Disabilities, 16(1), 51-74. doi:10.1016/0891-4222(94)00028-8.

Fleisher, D. Z., \& Zames, F. T. (2001). The Disability Rights Movement: From Charity to Confrontation. Philadelphia: University Press.

Fox, K. R., \& Corbin, C. B. (1989). The physical self-perception profile: development and preliminary validation. Journal of Sport and Exercise Psychology, 11, 408-30. doi:10.1123/jsep.11.4.408.

Gandek, B., Ware J.E., Aaronson N.K., Apolone G., Bjorner J.B., Brazier J.E., Bullinger M., Kaasa S., Leplege A., Prieto L., Sullivan M. (1998). Cross-validation of item selection and scoring for the SF-12 Health Survey in nine countries: results from the 
IQOLA Project. International Quality of Life Assessment. Journal of Clinical Epidemiology, 51(11), 1171-8.

Garcia, D., Nima, A. A., \& Kjell, O. N. E. (2014). The affective profiles, psychological well-being, and harmony: environmental mastery and self-acceptance predict the sense of a harmonious life. Peer J, 2, e259. doi:10.7717/peerj.259.

Gestsdottir, S., Magnusson, K., Arngrimsson, S. A., Johannsson, E., Arnarsson, A., \& Sveinsson, T. (2015). Gender differences in development of mental well-being from adolescence to young adulthood: an eight-year follow-up study. Scandinavian Journal of Public Health, 43(3), 269-75. doi:10.1177/1403494815569864.

Giacobbi, P. R. Jr., Stancil, M., Hardin, B., \& Bryant, L. (2008). Physical activity and quality of life experienced by highly active individuals with physical disabilities. Adapted Physical Activity Quarterly, 25(3), 189-207.

Groff, D. G., \& Zabriskie, R. B. (2006). An exploratory study of athletic identity among elite alpine skiers with physical disabilities: issues of measurement and design. Journal of Sport Behavior, 29(2), 126-41.

Grossi, E., Mosconi, P., Groth, N., Niero, M., \& Apolone, G. (2005). Questionario Psychological General Well Being Index. Versione Italiana. Milan: Istituto di Ricerche Farmacologiche "Mario Negri" and Bracco Imaging SpA.

Gundy, C. M., \& Aaronson, N. K. (2010). Effects of Mode of Administration (MOA) on the measurement properties of the EORTC QLQ-C30: a randomized study. Health and Quality of Life Outcomes, 8, 35. doi:10.1186/1477-7525-8-35.

Harada, S., Nishiwaki, Y., Michikawa, T., Kikuchi, Y., Iwasawa, S., Nakano, M., Ishigami, A., Saito, H., \& Takebayashi ,T. (2008). Gender difference in the relationships between vision and hearing impairments and negative well-being. Preventive Medicine, 47(4), 433-37. doi:10.1016/j.ypmed.2008.06.011 
Hood, K., Robling, M., Ingledew, D., Gillespie, D., Greene, G., Ivins, R., Russell, I., Sayers, A., Shaw, C., \& Williams, J. (2012). Mode of data elicitation, acquisition and response to surveys: a systematic review. Health Technology Assessment, 16(27), 1161. doi:10.3310/hta16270.

Janssen, I. (2007). Guidelines for physical activity in children and young people. Applied Physiology, Nutrition, and Metabolism, 32 Supp1 2, S122-35.

Jarvinen, T. A. H., Jozsa, L., Kannus, P., Jarvinen, T. L. N., \& Jarvinen, M. (2002). Organization and distribution of intramuscular connective tissue in normal and immobilized skeletal muscles. Journal of Muscle Research and Cell Motility, 23(3), $245-254$.

Kokaridas, D., Perkos, S., Harbalis, T., \& Koltsidas, E. (2009). Sport orientation and athletic identity of greek wheelchair basketball players. Perceptual and Motor Skills, 109(3), 887-98. doi:10.2466/pms.109.3.887-898.

Lankhorst, K., van der Ende-Kastelijn, K., de Groot, J., Zwinkels, M., Verschuren, O., Backx, F., Visser-Meily, A., Takken, T., \& HAYS study Group. (2015). Health in Adapted Youth Sports Study (HAYS): health effects of sports participation in children and adolescents with a chronic disease or physical disability. SpringerPlus, 4, 796. doi:10.1186/s40064-015-1589-z.

Li, R.-H., Kao, C.-M., \& Wu, Y.-Y. (2015). Gender differences in psychological wellbeing: tests of factorial invariance. Quality of Life Research, 24(11), 2577-81. doi:10.1007/s11136-015-0999-2.

Lundgren-Nilsson, Å., Jonsdottir, I. H., Ahlborg, G. jr, \& Tennant, A. (2013). Construct validity of the Psychological General Well Being Index (PGWBI) in a sample of patients undergoing treatment for stress-related exhaustion: a Rasch analysis. Health Qual. Life Outcomes, 11, 2. doi:10.1186/1477-7525-11-2 
Macdougall, H., O’Halloran, P., Sherry, E., \& Shields. N. (2016). Needs and strengths of australian para-athletes: identifying their subjective psychological, social, and physical health and well-being. The Sport Psychologist, 30(1), 1-12. doi:10.1123/tsp.2015-0006.

Martin, J. J., Adams-Mushett, C., \& Smith., K. L. (1995). Athletic identity and sport orientation of adolescent swimmers with disabilities. Adapted Physical Activity Quarterly, 12(2), 113-23.

Martin, J. J., Eklund, R. C., \& Mushet, C. A. (1997). Factor structure of the athletic identity measurement scale with athletes with disabilities. Adapted Physical Activity Quarterly, 14(1), 74-82. doi:10.1123/apaq.14.1.74.

Martin Ginis, K. A., Jetha, A., Mack, D. E., \& Hetz, S. (2010). Physical activity and subjective well-being among people with spinal cord injury: a meta-analysis. Spinal Cord, 48(1), 65-72. doi:10.1038/sc.2009.87.

Nieman, P. (2002). Psychosocial aspects of physical activity. Paediatrics and Child Health, 7, 309-12.

Nilsson, K. W., Leppert, J., Simonsson, B., \& Starrin, B. (2010). Sense of coherence and psychological well-being: improvement with age. Journal of Epidemiology and Community Health, 64(4), 347-52. doi:10.1136/jech.2008.081174.

Pack, S., Kelly, S., \& Arvinen-Barrow, M. (2016). "I think i became a swimmer rather than just someone with a disability swimming up and down" Paralympic athletes perceptions of self and identity development. Disability and Rehabilitation, Sep 27, 1-8. doi:10.1080/09638288.2016.1217074.

Pollard, E., \& Lee, P. (2003). Child well-being: a systematic review of the literature. Social Indicators Research, 61(1), 59-78. doi:10.1023/A:1021284215801.

Puce, L., Marinelli, L., Mori, L., Pallecchi, I., \& Trompetto, C. (2017). Protocol for the study of self-perceived psychological and emotional well-being of young paralympic 
athletes. Health and Quality of Life Outcomes, 15, 219. doi:10.1186/s12955-0170798-2.

Puce, L., Marinelli, L., Pierantozzi, E., Mori, L., Pallecchi, I., Bonifazi, M., Bove, M., Franchini, E., \& Trompetto, C. (2018). Training methods and analysis of races of a top level paralympic swimming athlete. Journal of Exercise Rehabilitation, 14(4): 612-20. doi:10.12965/jer.1836254.127.

Puhan, M. A., Ahuja, A., Van Natta, M. L., Ackatz, L. E., \& Meinert, C. (2011). Interviewer versus self-administered health-related quality of life questionnaires Does it matter? Health and Quality of Life Outcomes, 9, 30. doi:10.1186/1477-75259-30.

Ramos-Jorge, M. L., Vieira-Andrade, R. G., Martins-Júnior, P. A., Cordeiro, M. M. R., Ramos-Jorge, J., Paiva, S. M., \& Marques, L. S. (2012). Agreement between selfadministered and interviewer-administered CPQ 8-10 and CPQ 11-14. Community Dentistry and Oral Epidemiology, 40(3), 201-9. doi:10.1111/j.16000528.2011.00652.x.

Rasciute, S., \& Downward, P. (2010). Health or happiness? What is the impact of physical activity on the individual? Kyklos, 63, 256-270. doi:10.1111/j.14676435.2010.00472.x.

Rogers, A., Furler, B. L., Brinks, S., \& Darrah, J. (2008). A systematic review of the effectiveness of aerobic exercise interventions for children with cerebral palsy: an AACPDM evidence report. Developmental Medicine \& Child Neurology, 50(11), 808-14. doi:10.1111/j.1469-8749.2008.03134.x.

Runciman, P., Derman, W., Ferreira, S., Albertus-Kajee, Y., Tucker, R. (2015). A descriptive comparison of sprint cycling performance and neuromuscular characteristics in able-bodied athletes and Paralympic athletes with cerebral palsy. American Journal of Physical Medicine \& Rehabilitation, 94(1), 28-37. 
Runciman, P., Tucker, R., Ferreira, S., Albertus-Kajee, Y., Derman, W. (2016). Effects of induced volitional fatigue on sprint and jump performance in Paralympic athletes with cerebral palsy. American Journal of Physical Medicine \& Rehabilitation, 94(4), 277-90.

Rutherford, C., Nixon, J., Brown, J. M., Lamping, D. L., \& Cano, S. J. (2014). Using mixed methods to select optimal mode of administration for a patient-reported outcome instrument for people with pressure ulcers. BMC Medical Research Methodology, 14(1), 22. doi:10.1186/1471-2288-14-22.

Ryff, C., \& Keyes, C. (1995). The structure of psychological well-being revisited. Journal of Personality and Social Psychology, 69(4), 719-727. doi:10.1037/00223514.69.4.719.

Sawilowsky, S. S., (2009). New effect size rules of thumb. Journal of Modern Applied Statistical Methods, 8(2), 597 - 599. doi:10.22237/jmasm/1257035100.

Scully, D., Kremer, J., Meade, M. M., Graham, R., \& Dudgeon, K. (1998). Physical exercise and psychological well being: a critical review. British Journal of Sports Medicine, 32(2), 111-120.

Services, US Department of Health and Human. (2008). Physical activity guidelines advisory committee report.

Shephard, R.J. (1991). Benefits of sport and physical activity for the disabled: implications for the individual and for society. Scandinavian Journal of Rehabilitation Medicine, 23, 51-59.

Sherrill, C., Hinson, M., Gench, B., Kennedy, S. O., \& Low, L. (1990). Self-concepts of disabled youth athletes. Perceptual and Motor Skills, 70, 1093-1109.

Trompetto, C., Marinelli, L., Mori, L., Pelosin, E., Currà, A., Molfetta, L., \& Abbruzzese, G. (2014). Pathophysiology of spasticity: implications for neurorehabilitation. BioMed Research International, 2014, ID 354906. doi:10.1155/2014/354906. 
Tsakos, G., Bernabé, E., O’Brien, K., Sheiham, A., \& de Oliveira, C. (2008). Comparison of the self-administered and interviewer-administered modes of the child-OIDP. Health and Quality of Life Outcomes, 6, 40. doi:10.1186/1477-7525-6-40.

Verschuren, O., Ketelaar, M., Gorter, J. W., Helders, P. J., Uiterwaal, C. S., \& Takken, T. (2007). Exercise training program in children and adolescents with cerebral palsy: a randomized controlled trial. Archives of Pediatrics and Adolescent Medicine, 161(11), 1075-81.

Ware, J. E., Kosinski, M., \& Keller, S. D. (1996). A 12-item Short-Form health survey: construction of scales and preliminary tests of reliability and validity. Medical Care, 34, 220-33.

Ware, J. E., Kosinski, M. \& Keller, S. D. (1998). SF-12 how to score the SF-12 physical and mental health summary scales. Boston, Mass.: Quality Metric Inc., Boston, Mass., Health Assessment Lab.

Weinberger, M., Oddone, E. Z., Samsa, G. P., \& Landsman, P. B. (1996). Are healthrelated quality-of-life measures affected by the mode of administration? Journal of Clinical Epidemiology, 49(2), 135-40. doi:10.1016/0895-4356(95)00556-0.

Weiss, J., Diamond, T., Demark, J., \& Lovald, B. (2003). Involvement in Special Olympics and its relations to self-concept and actual competency in participants with developmental disabilities. Research in Developmental Disabilities, 24(4), 281-305. doi:10.1016/S0891-4222(03)00043-X.

Wely, L., Becher, J., Reinders-Messelink, H., Lindeman, E., Verschuren, O., Verheijden, J., \& Dallmeijer, A.J. (2010). LEARN 2 MOVE 7-12 years: a randomized controlled trial on the effects of a physical activity stimulation program in children with cerebral palsy. BMC Pediatrics, 10, 77. doi:10.1186/1471-2431-10-77.

Wiklund, I., \& Karlberg, J. (1991). Evaluation of quality of life in clinical trials: selecting quality of life measures. Controlled Clinical Trials, 12, S204-16. 
Wilson, P. E., \& Clayton, G. H. (2010). Sports and disability. $P M \& R$,. 2(3), S46-54. doi:10.1016/j.pmrj.2010.02.002.

Wright, J., \& Cowden, J. E. (1986). Changes in self concept and cardiovascular endurance of mentally retarded youths in Special Olympics swim training program. Adapted Physical Activity Quarterly, 3, 177-83. doi:10.1123/apaq.3.2.177.

Zwinkels, M., Verschuren, O., Lankhorst, K., van der Ende-Kastelijn, K., de Groot, J., Backx, F., Visser-Meily, A., Takken, T. \& Sport-2-Stay-Fit study Group. (2015). Sport-2-Stay-Fit study: health effects of after-school sport participation in children and adolescents with a chronic disease or physical disability. BMC Sports Science, Medicine and Rehabilitation, 7, 22. doi:10.1186/s13102-015-0016-7.

\section{Author Biographies}

Luca Puce (https://orcid.org/0000-0003-0825-2707) has a master degree in Sport Science and he is presently a PhD student in Neuroscience at the DINOGMI Department of the University of Genoa, Italy. His research activity is focused on technical sciences of preventive and adapted motor activity and on neurological disorders. He is also coach of a Paralympic swimming team, one member of which is a gold-medalist at the Rio 2016 Paralympic Games.

Lucio Marinelli (https://orcid.org/0000-0003-0620-7654) is an associate professor in Neurology at the University of Genoa, Italy. His research interests are mainly focused on the neural control of movement in patients with upper motorneuron disorders using a neurophysiological approach.

Nicola G. Girtler has a post degree in Clinical Psychology and Psychotherapy and he is presently a researcher in clinical psychology at the DINOGMI Department of the University of Genoa, Italy. He has continuously performed scientific research in the field of neuropsychology, neurophysiology, and clinical psychology especially about neurological disorders.

Ilaria Pallecchi (https://orcid.org/0000-0001-6819-6124) is a researcher in physics at the SPIN institute of the National Council of Researches. Besides her research activity on condensed matter physics, she is also interested in research on Paralympic sport. 
Laura Mori, $\mathrm{MD}, \mathrm{PhD}$, is an associate professor at the Department of Neurosciences, Rehabilitation, Ophthalmology, Genetics, Maternal and Child Health of University of Genoa and Physiatrist Physician affiliated with the National Health System at the IRCCS Ospedale Policlinico San Martino of Genoa. She has national and international scientific collaborations regarding different topics on neurorehabilitation. Particularly her interest are in developing and carrying out projects regarding Parkinson $\mathrm{s}$ disease, stroke, neuromuscular diseases, cerebellar ataxia, cognitive, swallowing, and motor recovery.

Marina Simonini is specialist in Physical Medicine and Rehabilitation. She is currently Director of the ASL 3 Genovese Rehabilitation Department. She is active in the research on motor control and movement analysis in acute and chronic neurological disabilities.

Carlo Trompetto is an associate professor of Physical Medicine and Rehabilitation at the DINOGMI Department of the University of Genoa. He is head of the Neuro-rehabilitation Unit of the University of Genova, Ospedale Policlinico San Martino-IRCCS. He is director of the School of Specialization in Physical Medicine and Rehabilitation, University of Genova. The principal areas of scientific interest are pathophysiology of upper motor ne neuron syndrome and movement disorders.

This manuscript has been published on Perceptual and Motor Skills Volume 126, Issue 5, October 2019, Pages 862-885, DOI 10.1177/0031512519865849

This post print version is released with a Creative Commons Attribution NonCommercial No Derivatives License, as required by Publisher's policy: http://sherpa.ac.uk/romeo/issn/0031-5125/ 